\title{
DIGESTIBILITÉ CHEZ LE PORC DES CONSTITUANTS D'UNE FARINE DE LUZERNE ARTIFICIELLEMENT DÉSHYDRATÉE.
}

PAR

G. CHARLET-LERY, A.-M. LEROY, S.-Z. ZELTER.

Laboratoire de Recherches Zootechniques. Institut National Agronomique, Paris.

Le régime alimentaire du porc comporte communément des céréales, des racines et des tubercules. L'apport azoté, minéral et vitaminique de ces aliments, produits sur l'exploitation ne suffit pas à satisfaire les besoins de cet animal. D'autre part, le processus digestif de celui-ci est essentiellement enzymatique, par conséquent peu apte à transformer des aliments cellulosiques dont la dégradation est liée à une intense activité bactérienne.

Or, les récents progrès accomplis en matière de déshydratation artificielle de fourrages verts permettent la production de farines d'herbes ou de légumineuses particulièrement riches en protides ainsi qu'en substances protectrices, et renfermant un faible pourcentage de cellulose.

Il était donc d'autant plus intéressant d'étudier l'utilisation digestive des constituants de la farine de luzerne déshydratée chez le porc, que les travaux sur ce sujet sont peu nombreux et que ce produit pourrait constituer, pour cette espèce animale, une source d'éléments nutritifs susceptibles de remplacer partiellement des aliments protidiques d'importation ou des farines d'origine animale relativement coûteuses.

Mitcheli et Hamilion (I) expérimentant sur un seul porc qui consomme une ration renfermant $27,5 \mathrm{p}$. Ioo d'une farine de luzerne très cellulosique $(29,26 \mathrm{p}$. Ioo), observent une digestibilité apparente de $42,7 \mathrm{p}$. Ioo pour la matière azotée, $67,4 \mathrm{p}$. Ioo pour les extractifs non azotés et $I, 8$ p. Ioo pour la cellulose brute. Chez le porc également, Richter (2) a trouvé, pour une luzerne récoltée en boutons et déshydratée, une digestibilité de 56 , o $\mathrm{p}$. Ioo pour la matière organique et 6r p. Ioo pour les protides totaux. Selon cet auteur (3) son de blé et farine de luzerne auraient, chez cet animal, des digestibilités voisines. Chez les ruminants, les valeurs correspondantes sont de 7 I et 78 p. roo. 
Plus récemment, Forbes et Hamiton (4) comparent l'utilisation de celluloses de différentes origines et enregistrent, chez le porc, pour un régime comportant environ $35 \mathrm{p}$. Ioo de farine de luzerne à $22 \mathrm{p}$. Ioo de cellulose brute, une digestibilité de 40,9 p. roo pour la cellulose brute, de $49,4 \mathrm{p}$. Ioo pour la cellulose vraie et de $74,9 \mathrm{p}$. roo pour l'azote. D'après ces chercheurs, la digestibilité de la cellulose de luzerne est nettement supérieure à celle de sciure de bois, de paille de blé ou de cuticules d'avoine.

Quant au taux de farine de luzerne que pourrait supporter le porc dans sa ration, les avis sont assez partagés. VAN VYK et VERBECH (5) ont remarqué que si le porc utilise bien des régimes contenant $34 \mathrm{p}$. Ioo de farine de luzerne, sa croissance s'en trouve cependant diminuée.

Bohman et coll. (6) trouvent peu de différence pour le gain de poids chez des porcs consommant de ro à $30 \mathrm{p}$. Ioo de farine de luzerne ; tandis que la croissance diminue d'environ $25 \mathrm{p}$. Ioo lorsque ce taux passe de 50 à 60 p. Ioo.

Une expérience plus récente $(7)$ durant laquelle ces mêmes auteurs ont donné des pourcentages de 0, Io, 30 et 50 de farine de luzerne montre de plus, que le rendement en viande et 1'épaisseur de lard dorsal diminuent avec l'accroissement du taux de luzerne tandis que la proportion de jambon et de longe dans la carcasse augmente significativement. D'après ces observations, le porc tolérerait sans inconvénient majeur jusqu'à $50 \mathrm{p}$. Ioo de farine de luzerne. L'influence heureuse de cet aliment sur la qualité des carcasses se trouve corroborée par les travaux de Crampton et coll. (8).

D'autres auteurs (9) considèrent cependant que le taux de farine de légumineuses devrait rester limité chez le porc entre 5 et I5 p. Ioo. GARD et coll. (Io), qui observent une dépression de croissance en présence de ro $\mathrm{p}$. Ioo de farine de luzerne déshydratée, partagent cette opinion.

Certains attribuent l'action dépressive sur la croissance aux saponines présentes en concentration très variable avec l'origine du fourrage ; Ces saponines constitueraient le facteur limitant d'utilisation et non la richesse en cellulose. En effet, des saponines extraites de ces farines et distribuées à des poussins ont provoqué une diminution considérable du gain de poids lorsque le taux de ces substances dans la ration a dépassé 0,20 p. IOO, taux correspondant à l'introduction de 25 p. roo du produit dont elle a été extraite (I2).

Ces données suggèrent qu'un taux de $35 \mathrm{p}$. Ioo de farine de luzerne est admissible dans le régime du porc; nous avons, en conséquence, étudié la digestibilité du produit en fixant le niveau expérimental maximum à ce seuil. 


\section{I. - Protocole expérimental :}

La farine de luzerne utilisée a été choisie parmi un lot de I8 échantillons qui ont été analysés au laboratoire (I2). Elle provenait d'une $3^{\text {e }}$ coupe de luzerne de I $^{\text {re }}$ année récoltée au début de septembre I953, dans le Pas-de-Calais et déshydratée dans un appareil Louisville (séchage à haute température, $800^{\circ} \mathrm{C}$ ).

Ses caractéristiques chimiques sont les suivantes, en p. mille de matière sèche :

Matières minérales : 137,4 ; matières organiques : 862,6 ; azote : 49,85; matières azotées: $(\mathrm{N} \times 6): 299, \mathrm{I}$; matières grasses : II7,9; cellulose Weende : I44, 8 dont environ 75 p. Ioo sous forme de cellulose Kurschner ; extractifs non azotés : 300,8; carotène : $315 \mathrm{mg}$.

Cette farine a été introduite successivement au taux de 20 et de $35 \mathrm{p}$. Ioo dans un régime de référence dont la digestibilité a été déterminée préalablement au cours d'une période témoin. Ce régime était constitué de : son d'avoine : $20 \mathrm{p}$. Ioo; orge : $52 \mathrm{p}$. Ioo; farine de viande : 6 p. Ioo; farine de poisson : 5 p. Ioo; levure de distillerie : 5 p. I0o; tourteau d'arachide : 8 p. Ioo ; mélange salin $\left(^{1}\right): 4$ p. Ioo. Sa matière sèche avait la composition chimique suivante, en grammes pour mille : matières minérales : 86,3 ; matières organiques : 913,7 ; azote : $35, \mathrm{r}$; matières azotées : 2 Io,6; matières grasses : 35,2 ; cellulose Weende : 84 , I ; extractifs non azotés : 572 , I.

Les 3 sujets d'expérience utilisés étaient des porcs Large White âgés de $3 \mathrm{I} / 2$ à 4 mois et pesant respectivement, au début de l'essai, 20,I-25,9 et $26,4 \mathrm{~kg}$. A la fin de celui-ci, leur âge et poids respectifs étaient de $6 \mathrm{I} / 2$ à 7 mois et de 58,66,2 et $70 \mathrm{~kg}$. Ils étaient exempts de parasites intestinaux.

Le type de cage de digestibilité, les méthodes de mesure et d'analyse chimique des ingesta et des excréta ont été décrits ailleurs (I3-I4).

Nous préciserons seulement que dans le présent essai, les périodes d'adaptation aux régimes expérimentaux ont duré au moins Io jours, et chacune des périodes expérimentales ro jours exactement.

Un seul type de ration a été expérimenté au cours de chaque période. Les types de régime étudiés se sont succédé comme suit :

$I^{0}$ période témoin : aliment de référence,

$2^{\circ}$ expérience $\mathrm{I}: 80 \mathrm{p}$. Ioo de matière sèche d'aliment de référence $+20 \mathrm{p}$. Ioo de matière sèche de farine de luzerne,

$3^{\circ}$ expérience $2: 65$ p. Ioo de matière sèche d'aliment de référence $+35 \mathrm{p}$. Ioo de matière sèche de farine de luzerne.

(1) Mélange salin en \%: poudre d'os dégélatiné : 40; craie lavée : $32 \%$; sel marin : 20 ; $\mathrm{SO}_{4} \mathrm{Mg}: 3,5 ; \mathrm{CO}_{3} \mathrm{Mg}: 4,2 ; \mathrm{SO}_{4} \mathrm{Fe}: 0,28 ; \mathrm{SO}_{4} \mathrm{Cu}: 0,02$. 
Les animaux ont été nourris ad libitum, mais le niveau de consommation de matière sèche a été maintenu pratiquement constant entre le milieu de la période d'adaptation et la fin de la période expérimentale. Les coefficients d'utilisation digestive des divers constituants de la farine de luzerne ont été calculés par la méthode dite de "différence ", d'après la composition chimique et la digestibilité des rations mixtes et du régime de référence.

\section{1. - Résultats et interprétation :}

\section{A. - Résultats :}

Les résultats sont condensés dans les tableaux I, II et III.

TABLEAU I

Composition des ingesta (en p. 1000. de matière sèche)

\begin{tabular}{|c|c|c|c|c|c|c|c|c|}
\hline & $\begin{array}{c}\text { Nature de } \\
\begin{array}{c}\text { Régime } \\
\text { de } \\
\text { référence }\end{array}\end{array}$ & $\underset{\substack{\text { Farine } \\
\text { de } \\
\text { luzerne }}}{\text { aliments }}$ & $\begin{array}{c}\text { Matières } \\
\text { minérales }\end{array}$ & $\begin{array}{c}\text { Matière } \\
\text { organique }\end{array}$ & Azote & $\begin{array}{c}\text { Matière } \\
\text { grasse }\end{array}$ & $\begin{array}{l}\text { Cellulose } \\
\text { Weende }\end{array}$ & $\begin{array}{c}\text { Extractifs } \\
\text { non } \\
\text { azotés }\end{array}$ \\
\hline $\begin{array}{l}\text { Période témoin.. } \\
\text { Expérience I... } \\
\text { Expérience } 2 . .\end{array}$ & $\begin{array}{r}\text { roo } \% \\
80 \% \\
65 \%\end{array}$ & $\begin{array}{l}\circ \\
20 \% \\
35 \%\end{array}$ & $\begin{array}{l}73,65 \\
\text { I01,80 } \\
\text { I03,6 }\end{array}$ & $\begin{array}{l}926,35 \\
898,2 \\
896,4\end{array}$ & $\begin{array}{l}35,3 \\
37,4 \\
39,35\end{array}$ & $\begin{array}{l}44,0 \\
57,7 \\
68,5\end{array}$ & $\begin{array}{r}81,7 \\
97,5 \\
1 \mathrm{I}, 7\end{array}$ & $\begin{array}{l}589,0 \\
518,6 \\
480, r\end{array}$ \\
\hline
\end{tabular}

\section{TABLEAU II}

Ingesta, Excréta et Coefficients d'utilisation digestive moyens des divers constituants des régimes.

\begin{tabular}{|c|c|c|c|c|c|c|c|}
\hline & $\begin{array}{l}\text { Matière } \\
\text { sèche }\end{array}$ & $\begin{array}{l}\text { Matières } \\
\text { minérales }\end{array}$ & $\begin{array}{l}\text { Matière } \\
\text { organique }\end{array}$ & Azote & $\begin{array}{c}\text { Matière } \\
\text { grasse }\end{array}$ & $\begin{array}{l}\text { Cellulose } \\
\text { Weende }\end{array}$ & $\begin{array}{c}\text { Extractifs } \\
\text { non } \\
\text { azotés }\end{array}$ \\
\hline & & & & & & & \\
\hline
\end{tabular}

Période témoin (régime de référence)

\begin{tabular}{|c|c|c|c|c|c|c|c|}
\hline Ingesta (g) & I 272,6 & 105,8 & І т 66,9 & 44,7 & 57,5 & 105,9 & 9 \\
\hline Excréta (g) & $4 I I, 6$ & 75,8 & 335,4 & IO,9 & 29,2 & 83,7 & 157,0 \\
\hline $\mathrm{CuD} \%$ & 67,7 & 28,6 & $7 \pm, 3$ & 75,6 & 49,2 & 21,0 & 78,6 \\
\hline
\end{tabular}

Expérience $I$ (régime à $20 \%$ farine de luzerne)

\begin{tabular}{|c|c|c|c|c|c|c|c|}
\hline Ingesta (g) & I 669,9 & 170,0 & I 499,9 & 62,4 & 94,4 & 162,9 & 865 \\
\hline Excréta (g) & $5 \circ 3,7$ & 92,3 & $4 \mathrm{II}, 5$ & 14,9 & 42,0 & 105,2 & I 75,0 \\
\hline $\mathrm{CuD}(\%)$ & 69,8 & 45,7 & 72,6 & $7^{6, I}$ & $5^{6,4}$ & 35,4 & \\
\hline
\end{tabular}

Expérience 2 (régime à $35 \%$ farine de luzeme)

\begin{tabular}{|c|c|c|c|c|c|c|c|}
\hline Ingesta $(\mathrm{g})$ & I 916,0 & I 98,4 & I 717,7 & 75,4 & I 3 I, 4 & $2 \mathrm{I} 4, \mathrm{I}$ & 919,7 \\
\hline Excréta $(g)$ & 630,5 & II 3,6 & $5 \mathbf{1} 6,8$ & $\mathrm{I} 9,4$ & 52 & 137,7 & 210,6 \\
\hline $\mathrm{CuD}(\%) \ldots$ & $67, \mathrm{I}$ & 84,8 & 69,9 & 74,2 & 60,4 & 35,7 & $77, \mathrm{I}$ \\
\hline
\end{tabular}




\section{Tableau III}

Coefficients individuels d'utilisation digestive (en p. 100) des constituants de la farine de luzerne (calculés par différence).

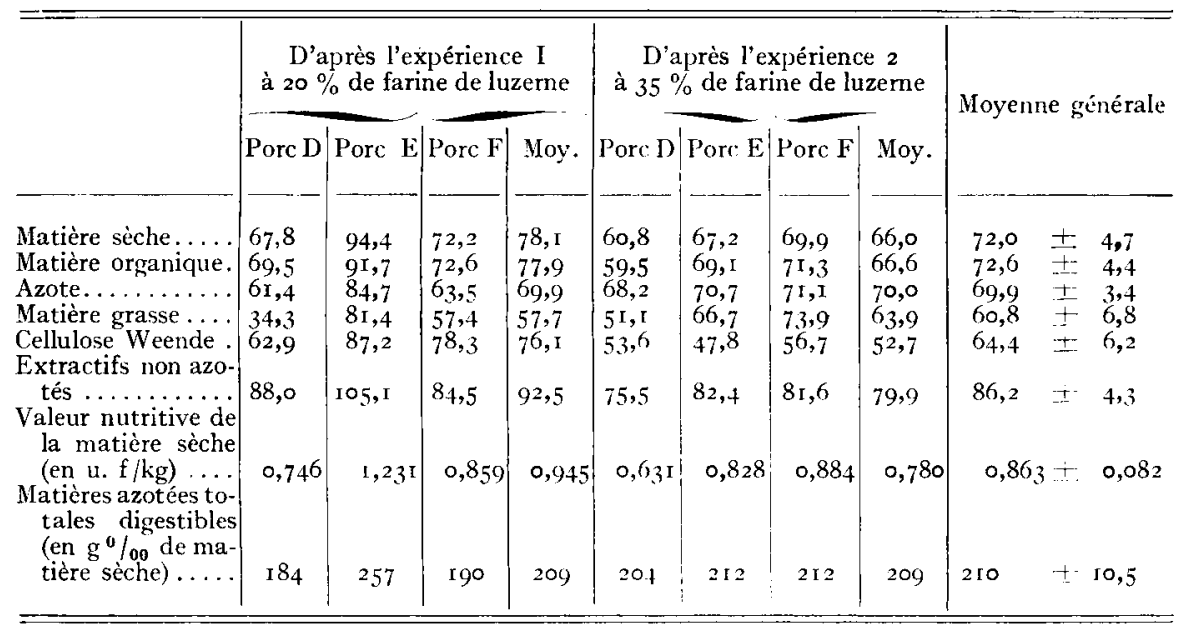

\section{B. - Interprétation :}

Signalons d'abord qu'au cours de l'expérience 2 avec le taux de 35 p. Ioo de luzerne, 1'appétence de la ration a diminué et les animaux ont eu tendance à restreindre légèrement, mais manifestement, leur consommation de matière sèche. De plus, des troubles digestifs se manifestant par des vomissements ont apparu vers la fin de l'essai. De tels symptômes n'ont pas été remarqués avec le taux de $20 \mathrm{p}$. Ioo; nous supposons que la richesse en saponines du produit expérimenté a pu être la cause de ce phénomène. Toutefois nous n'avons pas déterminé la teneur de notre farine de luzerne en ces substances, étant donnée l'imprécision actuelle des connaissances relatives à leurs structures et de leurs techniques de dosage.

Les données du tableau III montrent une différence d'utilisation digestive assez nette pour les deux pourcentages de farine de luzerne; cette utilisation est plus faible au taux de 35 qu'à celui de 20 p. Ioo. La diminution affecte en particulier les éléments cellulosiques, les extractifs non azotés et, par voie de conséquence, la matière organique, tandis que la digestibilité de la fraction protidique n'est pas modifiée. En passant de 20 à 35 p. Ioo, la chute de la valeur nutritive de la matière sèche atteint $-\mathrm{I} 7,5 \mathrm{p}$. Ioo.

Ces différences, qui ne sont pas élevées, autorisent le calcul de 
coefficients moyens de digestibilité pour chacun des constituants comme le prouvent, d'ailleurs, les écarts types de ces moyennes (').

Toutefois, si on compare les coefficients d'utlisation digestive du régime de référence et des régimes à 20 et $35 \mathrm{p}$. Ioo de farine de luzerne (tableau II), on constate que, malgré l'accroissement de la teneur en cellulose de la ration (tableau I) les variations sont extrêmement faibles. De ce fait, les valeurs nutritives des matières sèches de ces régimes $(0,8 \mathrm{I} 6$, 0,844 et 0,798 u. f. $/ \mathrm{kg}$ ) diffèrent, au maximum, de $5 \mathrm{p}$. Ioo.

Si donc on considère isolément la farine de luzerne, son utilisation paraît sensiblement meilleure à un taux de $20 \mathrm{p}$. Ioo ; mais, si l'on envisage dans son ensemble la ration dont elle fait partie, son introduction au taux de $35 \mathrm{p}$. Ioo affecte à peine l'utilisation nutritive de la matière sèche globale.

On peut par conséquent admettre que la farine de luzerne peut figurer sans inconvénient dans les rations pour porc en croissance et engraissement jusqu'à un taux de 35 p. Ioo, à la condition que sa teneur en saponines le permette.

Le tableau III fait ressortir, par ailleurs, des variations individuelles sensibles, surtout pendant l'expérience $I$ où le sujet $E$ a manifesté une utilisation digestive de la farine de luzerne, que l'on pourrait considérer comme aberrante (I,23I u. f./kg matière sèche). Ce même sujet accuse cependant au cours de l'expérience 2 des résultats comparables aux autres.

I,e calcul de la valeur nutritive (exprimée en unités fourragères) du $\mathrm{kg}$ de matière sèche de la farine de luzerne que nous avons étudiée et celui de la teneur en matières azotées totales digestibles avec et sans la donnée aberrante ci-dessus, donne les valeurs respectives suivantes; soit $0,863 \pm 0,082$ et 2 ro \pm ro, soit $0,790 \pm 0,046$ et $200 \pm 6$.

Ces données montrent donc que le porc utilise particulièrement bien une farine de luzerne contenant relativement peu d'éléments cellulosiques (I4-I5 p. IOO), même si elle figure à raison de $35 \mathrm{p}$. Ioo dans son régime. Sa richesse azotée la rend comparable à certaines catégories de tourteaux (coprah, palmiste) ; elle représente, par conséquent, une source très intéressante de protides végétaux pour cet animal, apportant en outre des substances protectrices telles que certains macro et micro éléments minéraux ainsi que des facteurs vitaminiques (provitamine $\mathrm{A}$, complexe B).

\section{III. - Résumé el Conclusions :}

L'utilisation digestive des constituants d'une farine de luzerne artificiellement déshydratée, expréssement choisie pour sa richesse en matières azotées totales $(29,9 \mathrm{p}$. roo) et sa faible teneur en cellulose

(1) Écart type $=\sqrt{\frac{\overline{\Sigma(x-\bar{x})^{2}}}{\mathrm{~N}(\mathrm{~N}-\mathrm{I})}}$. 
Weende (I 4,5 p. IOo) a été étudié sur 3 porcs par la technique dite "de différence ", au taux de 20 et $35 \mathrm{p}$. Ioo.

I,e résultat montre que, pour la farine en question, le taux de 35 p. Ioo constitue un seuil limite de tolérance dû probablement à la teneur en saponines.

Les coefficients de digestibilité des constituants sont plus élevés au niveau de $20 \mathrm{p}$. Ioo qu'à celui de $35 \mathrm{p}$. Ioo (dépression de valeur nutritive de - I7,5 p. Ioo). Toutefois, ce dernier taux affecte à peine l'utilisation nutritive de la matière sèche globale d'une ration équilibrée dans laquelle le produit a été introduit. La matière sèche de la farine de luzerne étudiée a une valeur nutritive de $0,790 \pm 0,046 \mathrm{u}$. f./kg, et une richesse en matière azotée totales, digestibles de $20 \pm 0,6$ p. Ioo.

\section{RÉFÉRENCES BIBLIOGRAPHIQUES}

(I) Mrtcheli (H. H.) et Hamiton (T. S.). - Digestibility of Oat Hulls and Alfalfa Meal by Swine. $J$. A gr. Res., I933, 4\%, no 6, p. 425.

(2) RichteR (K.). - Untersuchungen über den Futterwert Künstlich getrockneter Grünfuttermittel. Forschungsdienst, I94I, 10, 573.

(3) Richter (K.). - Die landwirtschaftliche Trocknung in ihrer Bedeutung für die Fütterung von Zucht- und Mastschweinen Vortrag Gesel. Landw. Trocknung, Sept. I952.

(4) Forbes (R. M.) et Hamilton ('T. S.). - The utilization of certain cellulosic materials by swine. J. Anim. Sci., I952, 11, no 3, 480 .

(5) VAN WYK et VERBECK. - I ucerne hay as a source of protein in the ration of baconer. Farming in South Africa, I95I, 26, 85.

(6) Bohman (V. R.), Kidwfil (J. F.), Mc Cormick (J. A.). - High levels of Alfalfa in the ration of growing, fattening swine. J. of Anim. Sci, I 953,12, p. 866.

(7) Bohman (V. R.), Hunter (J. E.), Mc Cormick (J. A.). - The effect of aureomycine upon utilization of graded levels of alfalfa by swine. J. Anim. Sci., 1954, 13, p. 977.

(8) Crampton (E. W.), Ashton (G. C.), Lloyd (L. E.). - Improvement of bacon carcass quality by the introduction of fibrous feeds into the hoy finishing ration. J. Anim. Sci., I954, 13, p. 227-33I.

(9) ELIIS (N. R.), ZELLER (J. H.), KING (J. X.). - The value of legume hays in the ration of fall farrowed pigs. J. Anim. Sci., I942, 1, p. 358.

(io) Gard (D. I.), Terrili (S. W.), Becker (D. E.), Norton (H. W.), NalBANDOV (A. V.). - Sources of unidentified factors for the pig. $J$. of Anim. Sci., I954, 13, p. 983 .

(II) Heywang (B. W.), BIRD (H. R.). - The effect of alfalfa saponin on the growth diet consomption and efficiency of diet utilization. of chicks. Poultry Science, I954, 33, p. 239.

(I2) LEROY (A.-M.), ZELTER (S.-Z.). - Composition et valeur alimentaire de quelques farines françaises de luzerne et de gazon déshydratés. Ann. de Zoot. (sous presse).

(I3) LEROY (A.-M.), LERY (G.), ZELTER (S.-Z.). - Contribution à l'étude de l'utilisation digestive des pulpes de betteraves desséchées par les porcs et les ruminants. Ann. de Zoot., I952, 1, n' ${ }^{\circ}$, p. 29.

(14) L,EROY (A.-M.), LERY (G.). - Utilisation des déchets de corne hydrolysés pour 1'alimentation du porc. Ann. Agron., I947, no 2, p. 264. 